\title{
Radiological surveillance of formerly asbestos- exposed power industry workers: rates and risk factors of benign changes on chest X-ray and MDCT
}

\author{
Christian Eisenhawer ${ }^{1 *}$, Michael K Felten ${ }^{1}$, Miriam Tamm², Marco Das ${ }^{3,4,5}$ and Thomas Kraus ${ }^{1}$
}

\begin{abstract}
Background: To determine the prevalence of asbestos-related changes on chest X-ray (CXR) and low-dose multidetector-row $\mathrm{CT}$ (MDCT) of the thorax in a cohort of formerly asbestos-exposed power industry workers and to assess the importance of common risk factors associated with specific radiological changes.

Methods: To assess the influence of selected risk factors (age, time since first exposure, exposure duration, cumulative exposure and pack years) on typical asbestos-related radiographic changes, we employed multiple logistic regression and receiver operating characteristic (ROC) analysis.

Results: On CXR, pleural changes and asbestosis were strongly associated with age, years since first exposure and exposure duration. The MDCT results showed an association between asbestosis and age and between plaques and exposure duration, years since first exposure and cumulative exposure. Parenchymal changes on CXR and MDCT, and diffuse pleural thickening on CXR were both associated with smoking. Using a cut-off of 55 years for age, 17 years for exposure duration and 28 years for latency, benign radiological changes in the cohort with CXR could be predicted with a sensitivity of $82.0 \%$ for all of the three variables and a specificity of $47.4 \%, 39.0 \%$ and $40.6 \%$, respectively.

Conclusions: Participants aged 55 years and older and those with an asbestos exposure of at least 17 years or 28 years since first exposure should be seen as having an increased risk of abnormal radiological findings. For implementing a more focused approach the routine use of low-dose MDCT rather than CXR at least for initial examinations would be justified.
\end{abstract}

Keywords: Asbestos, Power industry, Chest X-ray, Early detection, MDCT

\section{Background}

Asbestos is a naturally deposited fibrous mineral that was extensively mined and industrially used over many decades in the 20th century. Today the use of asbestos is largely restricted or banned in many industrialised countries because of well-known adverse health effects. Apart from the carcinogenic effect (lung cancer, laryngeal cancer and malignant pleural mesothelioma), exposure to asbestos dust can cause various benign changes of the lung (asbestosis) and the pleura (pleural plaques and diffuse pleural thickening) [1-5].

\footnotetext{
* Correspondence: ceisenhawer@ukaachen.de

${ }^{1}$ Institute of Occupational and Social Medicine, RWTH Aachen University, Aachen, Germany

Full list of author information is available at the end of the article
}

Statutory accident insurance institutions in Germany are legally required to actively detect clinical symptoms and conditions depending on occupational history, and to compensate asbestos-related diseases of lung, pleura and larynx. In the 1950s, the International Labour Organization (ILO) introduced an international classification of radiographs standardizing chest X-ray (CXR) reading of patients with pneumoconiosis [6-10]. Since the 1970s, the insurance institutions have therefore organised standardised surveillance programmes for the early detection of asbestosrelated diseases [4]. Already at that time, it was accepted that computed tomography had a higher sensitivity and specificity for the early detection of asbestos related changes of lung and pleura, although the required higher radiation dose in former times precluded a 
wider use in early detection programmes [11]. In spite of the rapidly declining industrial consumption of asbestos in Germany since the beginning of the 1980s, the number of patients at risk of asbestos-induced abnormal radiological findings continued to rise. The main reason for this was the long latency period of up to 40 years or more between first exposure and onset of disease, in combination with a still increasing asbestos consumption 40 years ago.

Reliable indicators of asbestos-induced benign parenchymal and pleural changes would be a very useful planning tool for setting up effective and affordable early detection programmes. Although CT technology has become more and more sensitive with smaller amounts of radiation used, early surveillance programmes in Germany are still generally based on conventional CXR [12-26]. Several reports on the use of MDCTs for early detection are available [27-33]. There is general agreement in the literature that the main factors influencing asbestos-related radiological changes are "age at examination" [13,26,27], "time since first exposure" (TSFE) [12,17-20,24,26,28,30,33], "years of exposure" [13,24,27,33], "cumulative asbestos exposure" $[13,15,17,19,22,25,27,31,33]$, and "smoking habits" [14,24]. High levels of cumulative exposure and history of smoking were usually shown to be associated with high rates of parenchymal changes. Time dependent factors such as age, TSFE and years of exposure were usually associated with pleural changes. In some studies cumulative exposure has been found to be associated with pleural plaques [34].

Thus the first aim of our study was to determine the prevalence of asbestos-related changes in a cohort of formerly asbestos-exposed power industry workers in two subgroups with different risk profiles using CXR (lower risk group) and MDCT (higher risk group). Our second aim was to assess the importance of common risk factors associated with specific radiological changes such as parenchymal fibrosis and pleural thickening. We did this with the view that determining rates of radiological changes typical for specific risk profiles would help differentiate large scale surveillance and early detection programmes.

\section{Methods}

\section{Cohort selection}

Enrolment for the survey was originally started in the late 1990s as a company internal programme of a major provider of electrical power in Germany. Main objective of this programme was the registration of asbestosexposed active and former employees. All persons were included in the cohort who could be contacted and who replied with a signed statement that they had been exposed to asbestos fibres. The individual cumulative exposure was determined by self-reported job titles and main occupational tasks. These data were analysed in a computer programme developed by occupational and safety experts and based on ambient monitoring data of airborne asbestos fibre concentrations at defined workplaces and the typical occupational tasks and time periods [35]. The cumulative asbestos exposure (in fibres/cubic centimetre $\times$ years) was expressed as the product of the eight-hour time weighted average fibre concentration and the total duration of exposure. One fibre year was defined as an exposure during 1920 work hours through daily eight-hour shifts over 240 workdays spread over 48 weeks with a standard airborne concentration of one fibre per cubic centimetre or $1 \times 10^{6}$ fibres per cubic metre.

Of the 5622 individuals who submitted a statement on asbestos exposure, we examined 4446 (79\%) during the study period.

The remaining 1176 were not examined for various reasons: contact too late or data incomplete: $44 \%$, refusal to participate: $21 \%$, appointment cancelled: $6 \%$, acute life-threatening disease: $0.6 \%$, deceased before intended examination: $12 \%$, no reply: $5 \%$, personal reasons: $7 \%$ and others: 4.4\% [36]. Another 42 examined participants had no radiological results because they refused any X-ray examination, or results of recently performed external examinations were not communicated. Of the remaining 4404 participants with valid CXR or CT results, we included only those with a complete set of data $(\mathrm{n}=3257,58 \%$ of the total cohort, CXR $\mathrm{n}=3061$, MDCT $\mathrm{n}=196)$ in the analysis. All participants were former or still active workers of a major provider of electrical power in Germany, most of them stationed in one of six power plants fired with lignite or coal. Most workers had been stationed in more than one plant during their career. Job characteristics were available for $94 \%(n=5284)$ of the total cohort indicating that $30 \%$ of them were metalworkers including welders, insulators and mechanics. Another 13\% were electricians, including communication technicians, and 30\% plant operators, including system controllers and boiler operators. $11 \%$ were employed as other craftsmen such as rooters, pipefitters, car mechanics, masons, gauge mechanics, turners, smelters, fire fighters, drivers, painters, warehousemen and forklift operators. A further $4 \%$ were present in the contaminated area but not conducting manual work for example work planners. $12 \%$ had other occupations such as security personnel, office staff or receptionists. More than half of the workers with data (59\% of 5284) were regularly and actively involved in revisions of steam turbines, $21 \%$ in removal of asbestos lagging and $4 \%$ in spraying of asbestos pulp. Another 36\% were involved in tooling and handling of asbestoscontaining gaskets, $30 \%$ in tooling packings, $26 \%$ in removal of asbestos mats, $28 \%$ in tooling gauges and $15 \%$ in maintenance of heat resistant wires [35]. Between March 2002 and the end of 2006 when the survey was closed for new admissions we invited the participants for standard 
medical examinations, including radiological examination of the thorax with CXR or MDCT.

Routine surveillance examinations were done in accordance with regulations by the German Social Accident Insurance (DGUV). Based on exposure history, smoking history and age at the time of examination, a subgroup of participants was considered as having an increased risk of developing asbestos-related radiologic changes. These participants were examined with MDCT [37]. CXR was not done in this subgroup. The rest of the cohort was examined by CXR (posterior anterior only). To classify the MDCT group, an empiric multiplicative risk calculation was used combining the factors exposure duration (years), $(\text { age } / 50)^{3}$ and smoking history (non-smoker $=0.1$, exsmoker $=0.3$, smoker $=1.0$ ). The resulting score was seen as a measure of the individual risk of developing asbestos-related radiological changes. A minimum score of 35 was required for admission to the MDCT subgroup.

The research obtained approval by the Institutional Review Board of the Medical Faculty, RWTH Aachen University (registration number EK2205).

\section{Screening protocol for MDCT}

In our study, MDCT examination of the whole lung in supine position during one breath-hold with deep inspiration without administration of contrast material was applied (SOMATOM Sensation 16, Siemens Medical Solutions, Forchheim, Germany). A standard low-dose MDCT protocol was used: $120 \mathrm{kV}, 10 \mathrm{mAs}_{\text {eff }}$ for individuals with less than $80 \mathrm{~kg}, 20 \mathrm{mAs}_{\text {eff }}$ for individuals with $80 \mathrm{~kg}$ and more, $16 \times 0,75 \mathrm{~mm}$ collimation, rotation time 0,5 seconds, table feed/rotation $18 \mathrm{~mm}$. Images were reconstructed in three different ways. The first stack of images was reconstructed with $5 \mathrm{~mm}$ effective slice thickness applying an increment of $4 \mathrm{~mm}$ with a medium smooth soft tissue convolution kernel (Siemens B30 kernel) window setting (center $\mathrm{C}=80$ $\mathrm{HU}$, window $\mathrm{W}=400 \mathrm{HU}$ ) for analysis of soft tissue changes, mediastinal changes and pleural changes. The next stack of images was reconstructed as $1 \mathrm{~mm}$ thick sections with a reconstruction increment of $0.5 \mathrm{~mm}$ and a sharp kernel (Siemens B50 kernel) $(C=-600$; $W=1500)$ for detection of pulmonary nodules, and the last stack of images was reconstructed as a high-resolution set with $1 \mathrm{~mm}$ thick sections every $10 \mathrm{~mm}$ with a B80 ultra-sharp reconstruction kernel $(C=-600 ; W=1500)$ for analysis of additional asbestos-related changes [37].

\section{Diagnosis of asbestosis and pleural changes on CXR and MDCT}

CXR was read according to the ILO 2000 classification [10]. A small opacity profusion of grade $1 / 1$ (or higher) at least in both lower fields was considered as a sign of asbestosis. Asbestos-related pleural change was defined as circumscribed (local) pleural thickening irrespective of location or dimension, or diffuse pleural thickening of grade " $2 \mathrm{a}$ " (or higher) at least in both middle and lower fields (according to the ILO classification). MDCT reading was based on the international CT-classification for pneumoconiosis, which has a similar format as the ILO classification for CXR reading $[9,38,39]$. We considered the following to be a sign of asbestosis on MDCT: bilateral interlobular septal thickening or intralobular lines (grade one or higher) at least in the lower fields, or parietal or visceral pleural changes irrespective of location and dimension.

The changes on CXR were basically grouped in those indicating any changes (parenchymal or pleural), asbestosrelated pulmonary fibrosis with or without pleural lesions (asbestosis) and plaques or diffuse pleural thickening with or without asbestosis. The reading results of the MDCTs were grouped in any changes (parenchymal or pleural), asbestosis with or without pleural lesions and pleural thickening (plaques) with or without asbestosis, including parietal and visceral changes (Table 1) $[10,40]$.

CXR and MDCT were read by two independent experienced readers. In cases of disagreement, a consensus reading was required. All readers were specialists in thoracic imaging, radiologists or specialists in occupational medicine.

\section{Statistical analysis}

For continuous variables mean and standard deviation as well as median, minimum and maximum are given. Categorical data are presented with frequencies and percentages.

Multiple logistic regression analysis was used to investigate the association of radiological changes with age, TSFE, years of exposure, cumulative exposure and pack years. Because of strong collinearities among the independent

Table 1 Number of participants with specific changes on standard chest X-ray $(n=3061)$ and MDCT of the thorax ( $n=196)$

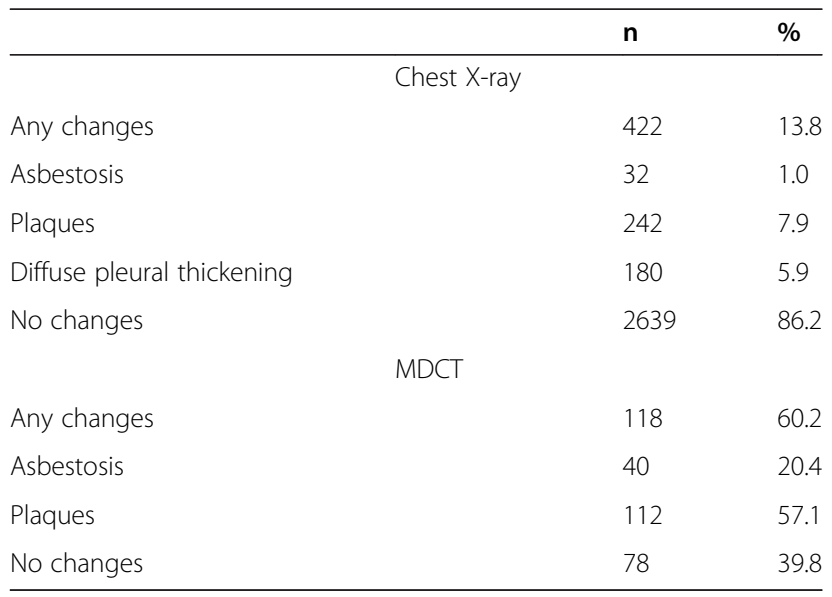


variables age (VIF $=3.0$ for the cohort with $\mathrm{CXR}, \mathrm{VIF}=1.3$ for the cohort with MDCT), TSFE (VIF = 8.2 for the cohort with CXR, VIF $=4.2$ for the cohort with MDCT) and years of exposure (variance inflation factor $[\mathrm{VIF}]=6.6$ for the cohort with CXR, VIF = 3.8 for the cohort with MDCT), separate models were fitted including burden of smoking (pack years), cumulative exposure (fibre years) and one of the three collinear variables. For the analysis of the fibre year values, we used three categories considering those with a calculated cumulative asbestos burden of less than or equal to one fibre year as negligibly exposed, as they had often been in contact with asbestos for only a few weeks. Because a fibre year value of 25 and more was a precondition for compensation payments in the German litigation systemfor lung cancer, we defined this as a cut-off value. We considered participants falling in the medium category with values between more than one and less than 25 years as notably exposed, but carrying only a limited additional asbestos-related risk of disease.

In order to determine the expected yield of health surveys among high-risk individuals for detecting asbestos-related abnormal radiological findings, we calculated receiver operating characteristic (ROC) curves. The ROC curves were used to determine the optimal cutpoint for age, TSFE and years of exposure to predict benign radiological changes when using standard examination with CXR. For the highrisk group with MDCT no cutpoints are given because the ROC curves yielded no acceptable discrimination (in all cases the area under the curve (AUC) does not exceed 0.6). To achieve an acceptable sensitivity, we decided to keep the sensitivity fixed in such a way that we attained a minimum sensitivity of $80 \%$ and maximized the specifity over the set of eligible cut-off values [41].

Particulary due to the large number of missing fibre year values for the variable cumulative asbestos exposure, the number of observations which could be used in the multiple logistic regression model was reduced to $n=3061$ for the subgroup with CXR examination and to $n=196$ for that with MDCT. To account for this fact, sensitivity analyses was done fitting the multiple regression models without the variable cumulative exposure so that $n=4158$ observations for the cohort with CXR examination and $\mathrm{n}=202$ observations for the cohort with MDCT were taken into account.

All applied tests were two-sided. Statistical analyses were performed in an explorative manner; thus p-values of $\mathrm{p} \leq 0.05$ could be interpreted as statistical significant test results.

ROC curves were calculated using the software MedCalc Version 11.4.4.0, Mariakerke, Belgium. All other analyses were carried out with the statistical software SAS Version 9.2, SAS Institute Inc., Cary, North Carolina, USA.

\section{Results}

Prevalence of asbestos-related radiological changes

The CXR group was characterised by low rates of both asbestos-related pulmonary changes (1\%) and pleural changes (plaques 7.9\%, diffuse pleural thickening 5.9\%) when compared to the corresponding rates found in the MDCT-group (20.4\% and 57.1\%). Table 1 shows the number of participants in the CXR and MDCT-groups with radiological changes typical for benign asbestosrelated findings of lung and pleura. While less than 15\% of the CXR-group showed abnormal radiological findings, two thirds (60.2\%) of the CT-group had typical changes. We explained the difference with the fact that the $\mathrm{CT}$ group had been preselected on the basis of a higher asbestos burden, and a correspondingly higher risk of disease, and the better performance of the MDCTs.

In the CXR group, we found only 32 cases of asbestosrelated changes of the lung (asbestosis), but a much higher rate of plaques $(n=242)$ and diffuse pleural thickening $(n=180)$. It was remarkable that the prevalence of diffuse pleural thickening and plaques was with 5.9\% and $7.9 \%$ quite similar. In the CT-group, the predominance of plaques $(n=112)$ was less pronounced with an only three-fold rate, possibly due to higher rates of prevalence and detection.

\section{Importance of risk factors}

Table 2 shows the prevalence of the five applied risk factors stratified by method of investigation (CXR or MDCT). As expected from the process of allocation to the two groups, the CT group had higher mean values for age, TSFE, years of exposure, cumulative exposure and pack years. Individuals

Table 2 Prevalence of applied risk factors stratified by method of investigation (chest X-ray or MDCT)

Mean Std. Dev. Median Minimum Maximum

\begin{tabular}{|c|c|c|c|c|c|}
\hline \multicolumn{6}{|l|}{ Chest X-ray } \\
\hline Age (years) & 57.0 & 11.5 & 58.0 & 27.0 & 90.0 \\
\hline TSFE $^{a}$ (years) & 32.4 & 10.9 & 31.0 & 3.0 & 65.0 \\
\hline $\begin{array}{l}\text { Exposure duration } \\
\text { (years) }\end{array}$ & 21.3 & 10.1 & 20.0 & $<1$ & 53.0 \\
\hline $\begin{array}{l}\text { Cumulative exposure } \\
\text { (fibre years) }\end{array}$ & 42.2 & 129.5 & 4.9 & $<1$ & 2331.4 \\
\hline Smoking (pack years) & 19.7 & 22.5 & 14.0 & 0 & 136.5 \\
\hline \multicolumn{6}{|l|}{ Low-dose MDCT } \\
\hline Age (years) & 65.8 & 6.1 & 65.5 & 41.0 & 84.0 \\
\hline TSFE $^{a}$ (years) & 41.2 & 7.4 & 41.0 & 20.0 & 57.0 \\
\hline $\begin{array}{l}\text { Exposure duration } \\
\text { (years) }\end{array}$ & 29.8 & 7.5 & 30.0 & 4.0 & 46.0 \\
\hline $\begin{array}{l}\text { Cumulative exposure } \\
\text { (fibre years) }\end{array}$ & 78.6 & 216.6 & 18.0 & $<1$ & 2558.2 \\
\hline Smoking (pack years) & 43.2 & 27.9 & 41.0 & 0 & 118 \\
\hline
\end{tabular}

atime since first exposure. 
with a high asbestos burden and those with only a minor exposure could be found in both subgroups.

The multiple logistic regression analysis of the CXR results indicated a significant effect of all considered risk factors (cumulative exposure only when comparing the groups with $\leq 1$ and $\geq 25$ fibre years) on the outcome changes. Signs of asbestosis on CXR were associated with all risk factors. However, we found an inverted effect of fibre year values on asbestosis (Table 3). The presence of plaques was mainly associated with age, TSFE and exposure duration. After adjusting for age, some effect could also be seen for cumulative exposure, especially when comparing the groups with less than or equal to one and 25 or more fibre years. Diffuse pleural thickening was associated with all risk factors including pack years, except cumulative exposure. When defining the variables age, TSFE and exposure duration as mainly time related and considering the variable cumulative exposure primarily as asbestos dose related, we can say that on CXR signs of asbestosis and diffuse pleural thickening were primarily associated with time whereas plaques were associated with both, time and exposure.

In the CT-group, the outcome variable of any changes was associated with TSFE and the cumulative exposure (considering the model adjusting for age). Signs of asbestosis were associated only with age and pack years, whereas pleural plaques were associated with TSFE, exposure duration and fibre years (group with $\leq 1$ versus $\geq 25$

Table 3 Changes on standard chest X-ray: results of multiple logistic regression model $(\mathrm{n}=3061$, odds ratios with $95 \%$ confidence interval and p-values of Wald test)

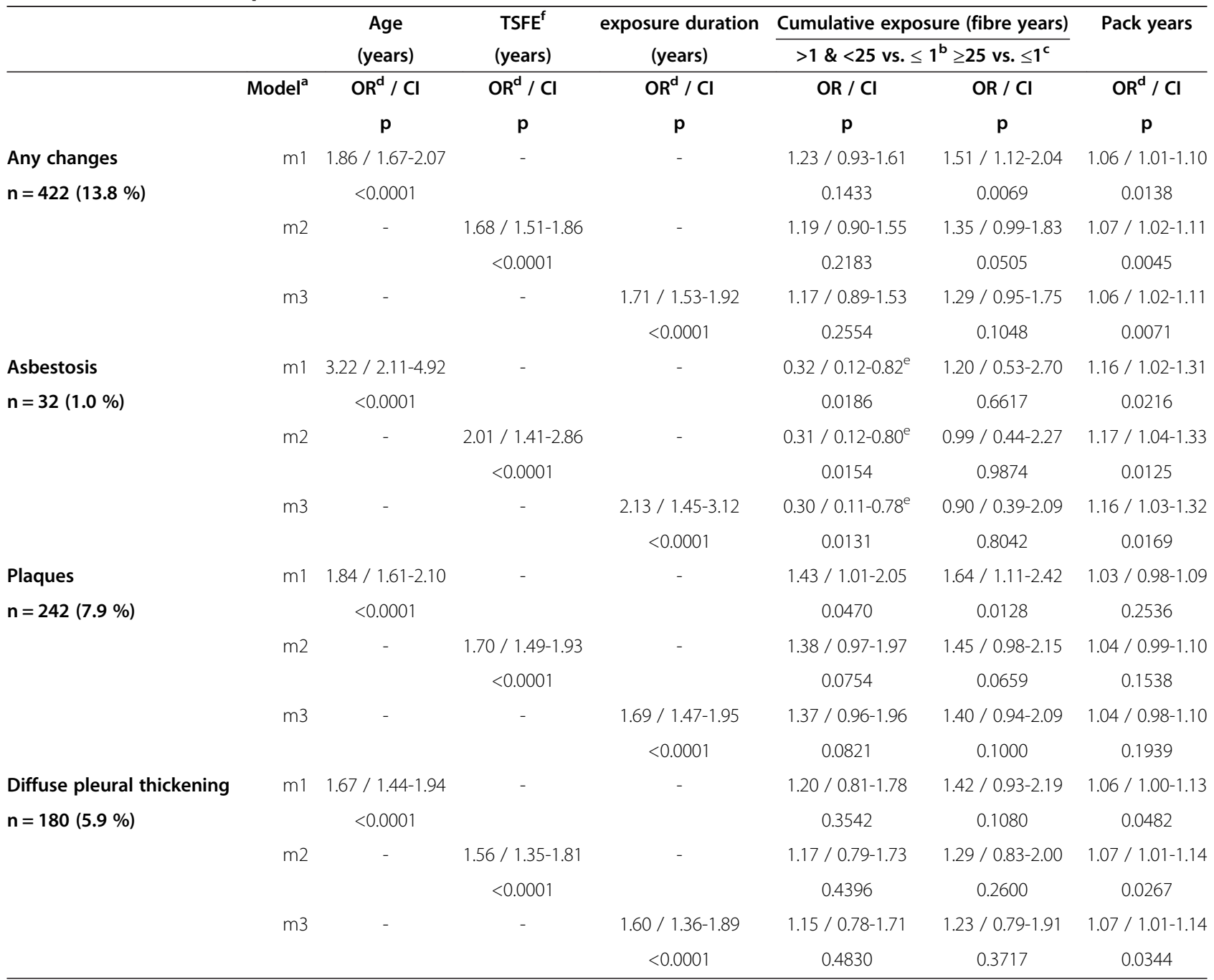

${ }^{a} \mathrm{~m} 1=$ model with age, $\mathrm{m} 2=$ model with latency, $\mathrm{m} 3=$ model with years of exposure.

${ }^{b}$ group with $>1$ and $<25$ fibre years versus group with $\leq 1$ fibre years.

cgroup with $\geq 25$ fibre years versus group with $\leq 1$ fibre years.

dodds ratio for an increase of 10 years (continuous variables).

eresults not plausible.

time since first exposure. 
fibre years, considering the model adjusting for age) (Table 4). Consistent with the CXR results, signs of asbestosis were only time-related and pleural lesions (plaques) were both time- and dose- related.

The results of the sensitivity analyses show that the statistical results in the multiple logistic regression were only marginaly affected by the reduction of number of observations due to the large number of missing values in the variable fibre years. In the CXR group the effect of pack years on diffuse pleural thickening was no longer significant. In the CT group age and years of exposure became significant for any changes and TSFE for asbestosis.

\section{ROC analysis}

Using ROC analysis to predict the outcome "any changes" with a minimum sensitivity of $80 \%$ and optimal specificity, we calculated in the CXR group cut-off values for age (55 years, area under the curve $(\mathrm{AUC})=0.694)$, TSFE (28 years, $\mathrm{AUC}=0.666)$ and exposure duration (17 years, AUC $=0.660$ ). With a sensitivity of $82 \%$ we obtained specificities of $47 \%, 41 \%$ and $39 \%$ for age TSFE and exposure duration. For the CT group we achieved no acceptable discrimination to develop good cut points (AUC $<0.6$ ), possibly due to the small sample size and the selection criteria applied for this group.

\section{Discussion}

Our cohort of 3257 asbestos-exposed workers had started their careers in the power industry when raw asbestos and asbestos-containing materials were routinely handled without effective protection.

The participants had been registered for a surveillance programme primarily designed for early detection of asbestos-related lung cancer. However, in the framework of our evaluation we did not consider cases of malignant asbestos-related diseases, but focused on benign asbestosrelated changes only. The type of the initial radiological examination was decided on the estimated risk of developing asbestos-related changes. As the allocation to the group with MDCTs was based on a high risk score (older participants with long exposure duration and extended smoking history), the two groups with MDCT and CXR were different in terms of age, exposure duration and smoking status (Table 2). This together with the different

Table 4 Changes on MDCT: results of multiple logistic regression model $(n=196$, odds ratios with $95 \%$ confidence interval, p-values of Wald test)

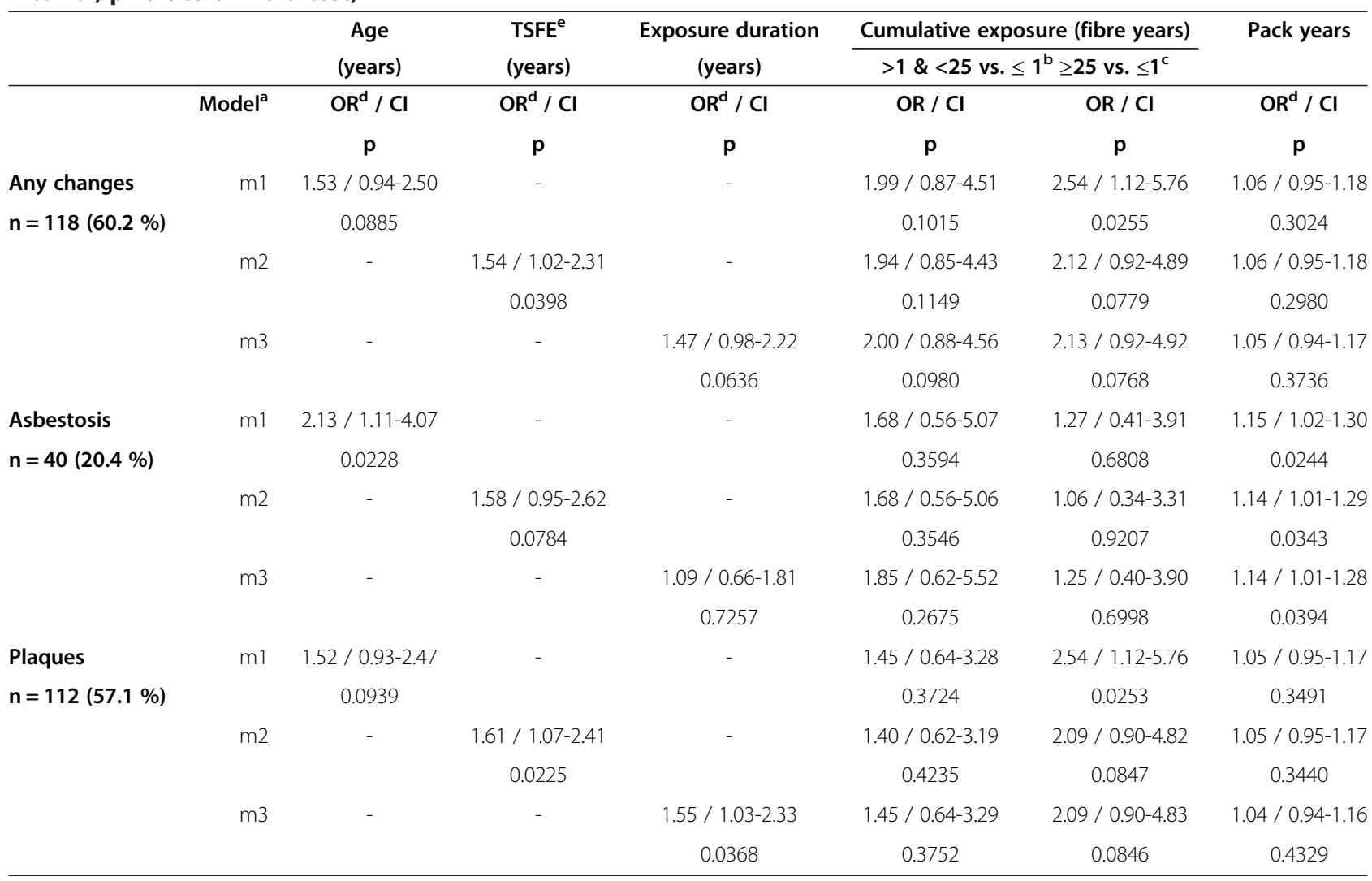

${ }^{a} \mathrm{~m} 1=$ model with age, $\mathrm{m} 2=$ model with latency, $\mathrm{m} 3=$ model with years of exposure.

${ }^{b}$ group with $>1$ and $<25$ fibre years versus group with $\leq 1$ fibre years.

cgroup with $\geq 25$ fibre years versus group with $\leq 1$ fibre years.

dodds ratio for an increase of 10 years (continuous variables).

${ }{ }_{\text {time since first exposure. }}$ 
reading routines made it necessary to consider both groups separately. We could still draw conclusions on the key role factors of radiological changes, which were reflected in the findings of both groups.

\section{Chest X-ray}

In CXR-based studies, an association between TSFE and the risk of developing pleural plaques is predominant. Jakobsson found a strong correlation between pleural plaques and TSFE, while the association with the cumulative asbestos exposure was much weaker [17]. The correlation between pleural plaques and TSFE was confirmed by Koskinen, who could also show some association with duration of exposure in a cohort of 18943 workers from construction industry, shipyards and asbestos industry in Finland [18]. Ehrlich found an exclusive correlation to TSFE in a multivariate analysis without any influence of cumulative exposure and exposure duration [19]. In a further study employing multivariate analysis, Matrat described a significant influence of TSFE for developing pleural thickening in 277 custodian and maintenance employees with generally low asbestos exposure [20]. In other studies, combined associations of age [26], duration of exposure, pack years and TSFE [24] were shown. Metintas described for a group of 991 villagers with environmental asbestos exposure in Turkey, an influence of age, gender, duration of exposure and type of asbestos on the development of local pleural thickening, whereas diffuse pleural thickening was influenced by age, duration of exposure and cumulative exposure [13].

Recent studies found that the cumulative exposure is the most important risk factor for the prevalence of asbestosis $[15,17,19,21-25]$. A correlation with smoking habits has been published [16,42]. In contrast Koskinen found a correlation to TSFE [18], Cookson [12] to TSFE and total exposure and Lilis [26] to TSFE and pack years. In our study, age, TSFE and duration of exposure and less pack years are significant risk factors for the prevalence of asbestosis. The inverse association between asbestosis and fibre year values $(\mathrm{OR}<1)$ did not seem plausible. These results are not corresponding with the most cited studies and may be the result of an effect of dose- related factors on the prevalence of asbestosis. We regard the low prevalence of asbestosis in CXR $(n=32)$ as one reason (see Table 1). As a second reason, the inverted association between asbestosis and fibre year values in the analysis of the group with $\leq 1$ fibre years versus the group with $>1$ and $<25$ fibre years was probably the result of misinterpretation of smoking-related changes in the lower fields as asbestosis. In the group with $\leq 1$ fibre years we found increased pack-year rates compared with the other groups, especially the group with $>1$ and $<25$ fibre years.

There was also a significant association between asbestosis and pack years as well as between diffuse pleural thickening and pack years (Table 3). In this regard, it is difficult to distinguish between asbestos and cigarette smoke-related changes on CXR examination in some cases. The statistical analysis of pleural changes in CXR in the largest group of plaques $(n=242)$ and in the group of diffuse pleural thickening $(n=180)$ shows a significant influence of age, exposure duration and TSFE as time-based risk factors, in accordance with the predominant literature. We also found a significant influence of cumulative exposure in the group of plaques. This result is not corresponding to the most cited studies. The low rate of asbestosis on CXR (1.0\%) and comparatively high rates of pleural changes (plaques $7.9 \%$, diffuse pleural thickening 5.9\%) was consistent with other published results (24). The expected predominance of diffuse pleural changes could not be shown, possibly due to the applied strict criteria together with the poor performance of the CXR in differentiating between the two pleural lesions. That may have led to readings of diffuse pleural thickening to be underestimated.

The ROC analysis results in optimal cut-offs of 55 years for age, 17 years for the exposure time and 38 years for latency to discriminate between patients with and without radiological chances. These cut-offs do not achieve an excellent discrimination as presented with the AUC. However, we decided to keep the sensitivity fixed, so that $82 \%$ of the patients of our cohort would be found to have radiological changes. It should be noted that the cut-off points are based on the particular cohort selected here for CXR. These would need to be validated in further studies.

\section{MDCT}

Paris noted a significant association between signs of fibrosis on high resolution CT (HRCT) and cumulative asbestos exposure in a cohort of 706 retired workers with a known occupational asbestos exposure in different industrial companies [27]. This study assessed the risk factors age, gender, smoking, duration of exposure and cumulative exposure index. The prevalence of pleural plaques was not examined in this study. In a further study of Paris, TSFE was the key variable for both, pleural plaques and asbestosis in a cohort of 1011 asbestos-exposed subjects in France [28]. Several parameters were calculated (TSFE, duration, intensity and cumulative exposure to asbestos and age). The cumulative exposure was also considered significant.

In a recent study using $\mathrm{HRCT}$, pleural plaques were associated with both TSFE $(\mathrm{p}<0.0001)$ and dose parameters (cumulative exposure $(\mathrm{p}=0.02)$ ) [33]. The prevalence of asbestosis was significantly associated only with dose parameters. No relationship was observed between duration of exposure and these two asbestos-related changes. The following risk factors were examined by 
logistic regression: TSFE, cumulative exposure index and intensity of exposure (adjusted for smoking status and body mass index). Schaeffner described a significant association between cumulative exposure index (level and duration of exposure) and simultaneous presence of pleural plaques and asbestosis [31]. TSFE was the main risk factor for both pleural plaques and asbestosis in a study of Algranti and lower association between asbestosis and cumulative exposure index [30]. In contrast, Cleemput could find no significant association between cumulative exposure or TSFE and the prevalence of pleural plaques [32]. In a recent study of Mastrangelo based on MDCT, the following variables were used in statistical analysis: Age, smoking habits, cumulative exposure, peak asbestos level, length of exposure and TSFE [29]. The results of the logistic regression models with stepwise selection of variables showed a significant association between cumulative exposure and asbestosis and TSFE and peak exposure and pleural plaques. TSFE was also correlated with diffuse pleural thickening.

In the analysis of MDCT, our results show a significant association between plaques, years of exposure and TFSE as time-based risk factors and cumulative exposure as a dose-based risk factor (Table 4). The association between asbestosis and cumulative exposure was described in several cited studies but not in our analysis. However, our cohort for MDCT was quite small with $\mathrm{n}=196$. For asbestosis, we found a significant association with age as time-based risk factor and pack years. In summary, we could show that time-based and dosebased risk factors were both associated with radiological changes indicating plaques. Parenchymal changes were associated primarily with the time-based factor age and corresponding to the results in our CXR-group with pack years as a sign of probable misinterpretation of smokingrelated changes. In contrast to several cited studies, dose-based risk factors such as exposure duration and cumulative exposure were not associated with parenchymal changes. Considering the low threshold we used for diagnosing asbestosis (bilateral interlobular septal thickening or intralobular lines with grade one or higher at least in the lower fields) and the observed significant association with pack years, we can interpret the results as partly indicating misclassification of irregular opacities in low profusion grades. The detected parenchymal changes were probably not a sign of asbestosis but rather nonspecific changes probably related to smoking.

In MDCT examination we found an increased prevalence of asbestosis $(n=40,20.4 \%)$ and plaques $(n=112$, 57.1\%) compared with CXR. The increased prevalence of asbestosis and pleural changes in MDCT examination can be explained by the higher sensitivity than CXR technology and the selection of this subgroup with the estimated higher duration of asbestos exposure.

\section{Conclusion}

We found a strong association between asbestos-induced radiological changes and the time-related risk factors age, TSFE and exposure duration. There was also a weak association between pleural lesions (CXR and MDCT) and cumulative exposure. Time-related factors had an overriding influence on most considered response variables excluding local plaques, which were also associated with the cumulative exposure. In comparable cohorts of asbestos exposed workers using a risk-dependent cohort selection, similar rates of benign asbestos-related changes of lung or pleura using CXR (14\%) or MDCT (60\%) can be expected.

In order to focus health surveillance efforts for asbestosrelated changes using CXR in asbestos-exposed workers, those aged 55 years and older should be examined with first priority. Alternatively, or after applying as a second criterion, those with an asbestos exposure of at least 17 years or with at least 28 years since first exposure should be screened. A more focused approach with a minimum number of individuals examined per case detected would also justify the routine use of MDCT rather than CXR for initial examination.

\section{Competing interests}

The authors declare that they have no competing interests.

\section{Authors' contributions}

CE coordinated the examination of participants, extracted the relevant data for analysis, interpreted the results and drafted the manuscript, MKF organized the cohort, managed the survey data and interpreted the results, TK conceived the study, designed the building of the cohort and the framework of the survey and interpreted the results. MT analysed the study data and supported their interpretation. MD evaluated the radiological method and data and revised them critically for important radiological content. All authors read and approved the final manuscript.

\section{Acknowledgements}

We express our gratitude to Thomas Küpper of the Institute of Occupational and Social Medicine for his support and advice when finalising the manuscript.

\section{Author details}

${ }^{1}$ Institute of Occupational and Social Medicine, RWTH Aachen University, Aachen, Germany. ${ }^{2}$ Department of Medical Statistics, RWTH Aachen University, Aachen, Germany. ${ }^{3}$ Department of Radiology, Maastricht University Medical Center, Maastricht, The Netherlands. ${ }^{4} \mathrm{GROW}$, School for Oncology \& Developmental Biology, Maastricht University Medical Center, Maastricht, The Netherlands. ${ }^{5}$ Department of Diagnostic Radiology, RWTH Aachen University, Aachen, Germany.

Received: 15 October 2013 Accepted: 17 April 2014

Published: 29 April 2014

\section{References}

1. Doll RPJ: Effects on Health of Exposure to Asbestos. Sudbury: Health and Safety Commission, HSE Books; 1985.

2. Tweedale G: The Rochdale asbestos cancer studies and the politics of epidemiology: what you see depends on where you sit. Int J Occup Environ Health 2007, 13:70-79.

3. Lin RT, Takahashi K, Karjalainen A, Hoshuyama T, Wilson D, Kameda T, Chan CC, Wen CP, Furuya S, Higashi T, Chien LC, Ohtaki M: Ecological association between asbestos-related diseases and historical asbestos consumption: an international analysis. Lancet 2007, 369:844-849. 
4. Hagemeyer $\mathrm{O}$, Otten $\mathrm{H}$, Kraus $\mathrm{T}$ : Asbestos consumption, asbestos exposure and asbestos-related occupational diseases in Germany. Int Arch Occup Environ Health 2006, 79:613-620

5. Tweedale G: Asbestos and its lethal legacy. Nat Rev Cancer 2002, 2:311-315.

6. Hering $K G$, Jacobsen $M$, Bosch-Galetke E, Elliehausen $H J$, Hieckel HG, Hofmann-Preiss K, Jacques W, Jeremie U, Kotschy-Lang N, Kraus T, Menze B, Raab W, Raithel HJ, Schneider WD, Strassburger K, Tuengerthal S, Woitowitz HJ, ILO: [Further development of the International Pneumoconiosis Classification-from ILO 1980 to ILO 2000 and to ILO 2000/German Federal Republic version]. Pneumologie 2003, 57:576-584.

7. Hering KG, Tuengerthal S, Kraus T: [Standardized CT/HRCT-classification of the German Federal Republic for work and environmental related thoracic diseases]. Radiologe 2004, 44:500-511.

8. Hering KG, Tuengerthal S, Kraus T, Wiebe V, Wegener HO, Raab W, Bohlig H: [CT study and standardised findings in occupationally-induced changes in the lungs and pleura with reference to the ILO 1980 classification of dust-induced pneumoconiosis]. Rontgenpraxis 1994, 47:262-269.

9. Kraus T, Raithel HJ, Lehnert G: Computer-assisted classification system for chest X-ray and computed tomography findings in occupational lung disease. Int Arch Occup Environ Health 1997, 69:482-486.

10. International Labour Organization: Guidelines for the use of the $1 / \mathrm{O}$ International Classification of Radiographs of Pneumoconioses 2000 edition. Geneva: International Labour Office; 2000.

11. Kraus T, Raithel HJ, Hering KG: Evaluation and classification of high-resolution computed tomographic findings in patients with pneumoconiosis. Int Arch Occup Environ Health 1996, 68:249-254.

12. Cookson WO, De Klerk NH, Musk AW, Armstrong BK, Glancy JJ, Hobbs MS: Prevalence of radiographic asbestosis in crocidolite miners and millers at Wittenoom, Western Australia. Br J Ind Med 1986, 43:450-457.

13. Metintas M, Metintas S, Hillerdal G, Ucgun I, Erginel S, Alatas F, Yildirim H: Nonmalignant pleural lesions due to environmental exposure to asbestos: a field-based, cross-sectional study. Eur Respir J 2005, 26:875-880.

14. Becklake MR: Asbestos and lung cancer: the clinicians' questions. Chest 1980, 78:372-373

15. Becklake MR: Asbestos and other fibre-related diseases of the lungs and pleura. Distribution and determinants in exposed populations. Chest 1991, 100:248-254

16. Ohar J, Sterling DA, Bleecker E, Donohue J: Changing patterns in asbestos-induced lung disease. Chest 2004, 125:744-753.

17. Jakobsson K, Stromberg U, Albin M, Welinder H, Hagmar L: Radiological changes in asbestos cement workers. Occup Environ Med 1995, 52:20-27.

18. Koskinen K, Zitting A, Tossavainen A, Rinne JP, Roto P, Kivekas J, Reijula K, Huuskonen MS: Radiographic abnormalities among Finnish construction, shipyard and asbestos industry workers. Scand J Work Environ Health 1998, 24:109-117.

19. Ehrlich R, Lilis R, Chan E, Nicholson WJ, Selikoff IJ: Long term radiological effects of short term exposure to amosite asbestos among factory workers. Br J Ind Med 1992, 49:268-275.

20. Matrat M, Pairon JC, Paolillo AG, Joly N, Iwatsubo Y, Orlowski E, Letourneux M, Ameille J: Asbestos exposure and radiological abnormalities among maintenance and custodian workers in buildings with friable asbestos-containing materials. Int Arch Occup Environ Health 2004 77:307-312.

21. Sluis-Cremer GK, Hessel PA, Hnizdo E: Factors influencing the reading of small irregular opacities in a radiological survey of asbestos miners in South Africa. Arch Environ Health 1989, 44:237-243.

22. Copes R, Thomas D, Becklake MR: Temporal patterns of exposure and nonmalignant pulmonary abnormality in Quebec chrysotile workers. Arch Environ Health 1985, 40:80-87.

23. Finkelstein MM, Vingilis JJ: Radiographic abnormalities among asbestos-cement workers. An exposure-response study. Am Rev Respir Dis 1984, 129:17-22

24. Jones RN, McLoud T, Rockoff SD: The radiographic pleural abnormalities in asbestos exposure: relationship to physiologic abnormalities. J Thorac Imaging 1988, 3:57-66.

25. Stayner L, Smith R, Bailer J, Gilbert S, Steenland K, Dement J, Brown D, Lemen R: Exposure-response analysis of risk of respiratory disease associated with occupational exposure to chrysotile asbestos. Occup Environ Med 1997, 54:646-652.

26. Lilis R, Miller A, Godbold J, Chan E, Selikoff IJ: Radiographic abnormalities in asbestos insulators: effects of duration from onset of exposure and smoking. Relationships of dyspnea with parenchymal and pleural fibrosis. Am J Ind Med 1991, 20:1-15.

27. Paris C, Benichou J, Raffaelli C, Genevois A, Fournier L, Menard G, Broessel N, Ameille J, Brochard P, Gillon JC, Gislard A, Letourneux M: Factors associated with early-stage pulmonary fibrosis as determined by high-resolution computed tomography among persons occupationally exposed to asbestos. Scand J Work Environ Health 2004, 30:206-214.

28. Paris C, Martin A, Letourneux M, Wild P: Modelling prevalence and incidence of fibrosis and pleural plaques in asbestos-exposed populations for screening and follow-up: a cross-sectional study. Environ Health 2008, 7:30.

29. Mastrangelo G, Ballarin MN, Bellini E, Bicciato F, Zannol F, Gioffre F, Zedde A, Tessadri G, Fedeli U, Valentini F, Scoizzato L, Marangi G, Lange JH: Asbestos exposure and benign asbestos diseases in 772 formerly exposed workers: dose-response relationships. Am J Ind Med 2009, 52:596-602.

30. Algranti E, Mendonca EM, DeCapitani EM, Freitas JB, Silva HC, Bussacos MA: Non-malignant asbestos-related diseases in Brazilian asbestos-cement workers. Am J Ind Med 2001, 40:240-254.

31. Schaeffner ES, Miller DP, Wain JC, Christiani DC: Use of an asbestos exposure score and the presence of pleural and parenchyma abnormalities in a lung cancer case series. Int J Occup Environ Health 2001, 7:14-18

32. Van Cleemput J, De Raeve H, Verschakelen JA, Rombouts J, Lacquet LM, Nemery B: Surface of localized pleural plaques quantitated by computed tomography scanning: no relation with cumulative asbestos exposure and no effect on lung function. Am J Respir Crit Care Med 2001, 163:705-710.

33. Paris C, Thierry S, Brochard P, Letourneux M, Schorle E, Stoufflet A, Ameille J, Conso F, Pairon JC: Pleural plaques and asbestosis: dose- and time-response relationships based on HRCT data. Eur Respir J 2009, 34:72-79.

34. Jarvholm B: Pleural plaques and exposure to asbestos: a mathematical model. Int J Epidemiol 1992, 21:1180-1184.

35. Felten MK, Knoll L, Eisenhawer C, Ackermann D, Khatab K, Hudepohl J, Zschiesche W, Kraus T: Retrospective exposure assessment to airborne asbestos among power industry workers. J Occup Med Toxicol 2010, 5:15

36. Knoll L, Felten MK, Ackermann D, Kraus T: Non-response bias in a surveillance program for asbestos-related lung cancer. $J$ Occup Health 2011, 53:16-22.

37. Das M, Muhlenbruch G, Mahnken AH, Hering KG, Sirbu H, Zschiesche W, Knoll L, Felten MK, Kraus T, Gunther RW, Wildberger JE: Asbestos Surveillance Program Aachen (ASPA): initial results from baseline screening for lung cancer in asbestos-exposed high-risk individuals using low-dose multidetector-row CT. Eur Radio/ 2007, 17:1193-1199.

38. Kraus T, Borsch-Galetke E, Elliehausen HJ, Frank K, Hering KG, Hieckel HG, Hofmann-Preiss K, Jacques W, Jeremie U, Kotschy-Lang N, Mannes E, Otten H, Raab W, Raithel HJ, Schneider WD, Tuengerthal S: [Recommendations for reporting benign asbestos-related findings in chest X-ray and CT to the accident insurances]. Pneumologie 2009, 63:726-732.

39. Kraus T, Borsch-Galetke E, Elliehausen HJ, Frank K, Hering KG, Hieckel HG, Hofmann-Preiss K, Jacques W, Jeremie U, Kotschy-Lang N, Mannes E, Otten H, Raab W, Raithel HJ, Schneider WD, Tuengerthal S: [Examples for asbestos-related findings in HRCT - criteria for the assessment of causal relationships in surveillance programmes and medical expert opinion]. Pneumologie 2010, 64:37-44.

40. AmericanThoracicSociety: Diagnosis and initial management of nonmalignant diseases related to asbestos. Am J Respir Crit Care Med 2004, 170:691-715.

41. David LRBH, Guyatt GH, Tugwell P: Clinical Epidemiology a Basic Science for Clinical Medicine (2nd Edition). Boston: Little Brown and Company; 1991.

42. Becklake MR, Arhirii MI, Gibbs GW, Hurwitz S: Exposure to asbestos and respiratory abnormality: the influence of fibre type and nature of exposure. IARC Sci Pub/ 1980, 30:763-768.

doi:10.1186/1745-6673-9-18

Cite this article as: Eisenhawer et al:: Radiological surveillance of formerly asbestos-exposed power industry workers: rates and risk factors of benign changes on chest X-ray and MDCT. Journal of Occupational Medicine and Toxicology 2014 9:18. 